\title{
Dynamic Traffic Load Balancing Mechanism for SHAKE Architecture
}

\author{
Hiroshi Esaki, \\ Hiroki Kan \\ Graduate School of Information Science and Technology, The University of Tokyo, Japan \\ hiroshi@wide.ad.jp \\ kohki@hongo.wide.ad.jp
}

\begin{abstract}
In the mobile ad-hoc network, we frequently experience the case where only the small bandwidth cellpone links are available without sharing these expensive bandwidth's, while high bandwidth wireless LAN link is available among end-stations. SHAKE, SHAred multiple paths protocol for cluster networK, is the architecture using the mobile IP so as to share the multiple external cell-phone links via the wireless LAN link. Since the traffic load balancing in the existing SHAKE system is of static, the actual available bandwidth for the end-station goes to bad performance. This is because the available bandwidth for each cell-phone links is not stable and dynamically changed.

In this paper, the dynamic traffic balancing mechanism for SHAKE system with available bandwidth measurement is proposed and is proposed and performed a preminary evaluation. The prototype system implementing the proposed mechanism can achieve large performance improvement.
\end{abstract}

\section{Introduction}

High speed 3G cell-phone-card and PHS-data-card provide a large bandwidth link to access the Internet for mobile end-stations. However, comparing to the available bandwidth provided by WiFi system, it is far smaller. Though the bandwidth provided by WiFi system can be shared among the end-stations on the same link, the bandwidth's of each cell-phone-card or PHS-data-card can not be shared among the end-stations.

In the ad-hoc network environment, we frequently experience the case where the high speed wired connectivity via WiFi link is not available, while some small bandwidth cell-phone or PHS links provided by the end-stations are available. This is not a disconnected adhoc network, but is the network has multiple exclusively available small bandwidth connectivity's for each individual mobile end-station. In order to obtain the larger available bandwidth, the end-station wants to aggregate the external links that could be offered by the end-stations with their small bandwidth external links, and these endstations must work as router, instead of as host. This (ad- hoc) network is so called as a multi-homing network, which has multiple network connectivity's to the different network providers. There are many architectural proposals to achieve multi-homing.

SHAKE [1], SHAred multiple paths protocol for cluster networK, is one of the architecture for multi-homing, using the mobile IP[2]. In SHAKE, the mobile IP operation is used to share the multiple external cell-phone links provided by co-operative end-stations. These cooperative end-stations are connected to each other via the high bandwidth WiFi link.

However, since the traffic load balancing in the existing SHAKE system is of static based on the link bit-rate of the external link, the actual available bandwidth for the endstation goes to bad performance. This is because the available bandwidth for each cell-phone links is not stable, is dynamically changed and is different from the link bitrate. The link bit-rate and the available bandwidth are always not identical. In this paper, the dynamic traffic balancing mechanism for SHAKE system with the available bandwidth measurement is proposed and evaluated. The prototype system implementing the proposed mechanism shows that the proposed system can achieve large performance improvement

Section 2 describes the SHAKE and related works. Section 3 describes the proposed mechanisms, i.e., available bandwidth measurement scheme and load balancing, to improve the performance of existing SHAKE system. Section 4 describes the prototype implementation and it's performance evaluation. Finally, section 5 gives brief summary of this paper.

\section{Related Works}

In this section, the related works with this paper are presented.

\subsection{Mobile IP [2]}

In a legacy network, an IP address belonging to the corresponding physical network segment is allocated. When a node moves to the other network segment, it's IP address will be changed and the node can not preserve the TCP connections without applying some mechanisms, such as SCTP[3], parallel TCP[4], M/TCP[5] or mobile IP. 
Here, we call the node, which change the location, as MN(Mobile Node). Mobile IP can preserve all the TCP connections, even when an MN moves from some segment to the other segment. This is because the source IP address of every TCP connections will not be changed, due to the change of network segment where the node belongs to. Of course, it is the case where the disconnected period, due to location change, is short enough to avoid the termination of TCP connection by the expiration of TCP's persistent timer.

$\mathrm{MN}$ has multiple IP addresses, i.e., HoA(Home Address) and $\mathrm{CoA}$ (Care of Address). HoA is used to establish the TCP connection with the corresponding destination nodes. When $\mathrm{MN}$ is away from the network segment where HA belongs to, HA(Home Agent) is the node intercepting the IP packets, that are forwarded to the $\mathrm{MN}$, and forward these IP packets toward the mobile node having the corresponding HoA using the IP-in-IP tunneling [6]. HA advertises the routing information, which includes all the IP address of MNs taken care of by the corresponding HA, the Internet. In order to intercept the IP packets to the particular MNs, that are away from the home network, every MN being away from it's home network performs the registration of CoA to HA.

\subsection{Multi-homing with MIP and NEMO Framework [7][8][9]}

Multi-homing and multi-path technology improves the availability of host and network connectivity. Since the node and network behavior of mobile networking and static networking are different, the different architecture has been discussed and proposed. This architecture provides the common architecture both for mobile and static networking environment, using the MIP and NEMO(Network Mobility) [8] framework. The proposed architecture only requires (1) modification of Mobile IP so that multiple-CoA can be used, (2) policy control protocol for multiple-path packet transmission.

Paper [7] and [9] propose the concept of "virtual" home network for nodes. We do not need the physical home network for MNs or mobile network. Multiple HAs should be geographically distributed across the Internet, for the improvement of service availability and for the load balancing/distribution. When all the multiple HAs advertise the same network prefix to their adjacent router/network, the traffic is automatically routed to the nearest HA from the view point of routing protocol topology. Multiple tunneling paths between HA and multi-homed node (host/router) should be able to be established.

\subsection{SHAKE [1]}

SHAKE is the architecture to share the small bandwidth external links among the end-stations, which are connected via a large bandwidth local link such as WiFi. From the view point of an end-station, the multiple external links can be aggregated in order to increase the available bandwidth so as to communicate with any node in the global Internet space. It is said that, by using the multiple external links, the improvement of communication qualities, associated with bandwidth, latency and robustness, could be achieved. It has been proposed three types of SHAKE implementations. These are (1) Web SHAKE implemented at application layer, (2) TCP SHAKE implemented at TCP layer like SCTP, and (3) IP SHAKE implemented at IP layer with MIP.

In this paper, we discuss about IP SHAKE with MIP, that is called as Mobile IP SHAKE. In this architecture, HA works as the load balancer to use multiple paths toward the destination node (i.e., MN). Figure 1 and 2 show the architecture and IP packet transmission in Mobile IP SHAKE system.

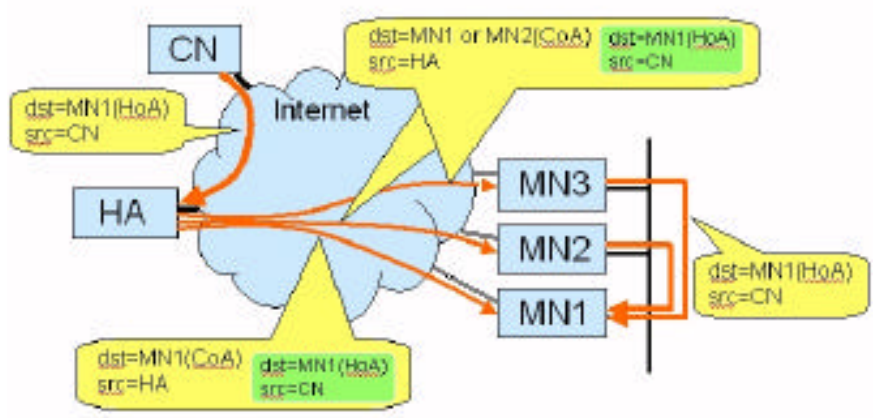

Figure 1. IP packet transmission from $\mathrm{CN}$ to $\mathrm{MN}$

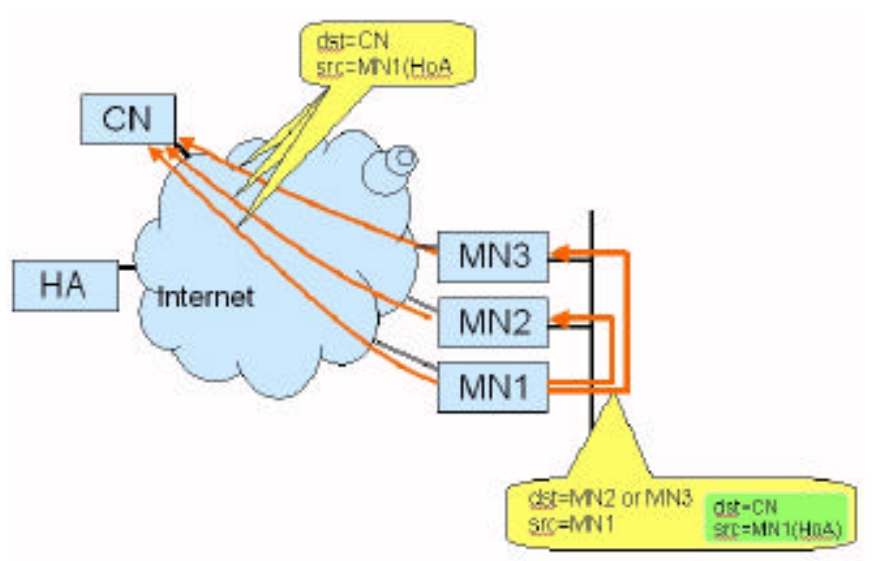

Figure 2 IP packet transmission from MN1 to CN

As shown in figure 1 and 2, mobile nodes (MN2 and MN3 in the figures) allow to use their bandwidth by MN1. MN2 and MN3 work as a kind of router, i.e., forward the 
received IP packets, and take care of IP tunneling. The IP packets from $\mathrm{CN}$ to $\mathrm{MN} 1$ are distributed into three paths at HA. The IP packets from MN1 to $\mathrm{CN}$ are distributed into three paths by MN1.

The algorithms of IP packets distribution implemented in the existing Mobile IP SHAKE are a simple round robin and the weighted round robin proportional to the links' bit-rate.

- Simple round robin

All paths are equally used. When the latency diversity and/or the available bandwidth among the multiple paths is large enough, the system experiences serious performance degradation. The performance degradation will occur due to the packet re-ordering observed at the destination node (MN1), and the performance is suffered from the worst quality and the smallest available bandwidth path.

- Weighted round robin proportional to the links' bitrate

IP packets are distributed in proportional to the links' bit-rate. When the latency is the same among the multiple paths and the available bandwidths' are actually proportional to the corresponding link bitrate, the system should achieve good performance. However, in general, the latency among the multiple paths are not identical, and the actually available bandwidth versus it's link bit-rate is not identical among multiple paths and it is changed according the network status.

\section{Proposed Traffic Distribution Mechanism for SHAKE System}

In this section, the mechanisms of the traffic distribution so as to improve the actual available bandwidth performance of IP packet delivery in the existing SHAKE system.

As discussed in the previous section, the existing SHAKE system applies the simple weighted round robin based on link's bit-rate. However, in the actual system, the available bandwidth would not proportional to the corresponding link bit-rate, and the available bandwidth is changed according to the network condition.

We propose the IP packet distribution mechanism, based on the available bandwidth measurement for each alternative path. The IP packets are distributed at HA, in proportional to the measured available bandwidth. Here, since the external links do not have large bandwidth, we have to carefully choose the bandwidth estimation mechanism. Especially with the actual measurement mechanism, the accuracy of measurement and the requires traffic volume for bandwidth measurement is generally of trade-off parameters.

\subsection{Available bandwidth measurement}

In this subsection, we discuss and propose the mechanism for available bandwidth to be applied in the proposed system.

- $\quad$ One way path bandwidth measurement

Since an MN has multiple up-stream paths (from MN to $\mathrm{CN}$ ) and down-stream paths (from $\mathrm{CN}$ to $\mathrm{MN}$ via HA), we have to measure all of these uni-directional paths. We could measure the bandwidth against the round-trip path. However, since the available bandwidth for up-stream and down-stream is not generally symmetric, the bandwidth measurement should be for one way path.

- Bandwidth measurement by packet pair

There are passive and active for bandwidth measurement. With passive measurement, we have to analyze the bandwidth using the user packets. User packets would not be always transmitted from the nodes, and it would not be easy to obtain the available bandwidth with small latency. On the contrary, with active measurement, we generate the probe packets to measure the bandwidth. Though we must waste the bandwidth by these probe packets, it would be easier to obtain accurate results with small latency. Therefore, we propose to use the active measurement in the proposed system. There are a lot of active measurement mechanisms for available bandwidth. With the consideration from the view point of bandwidth consumption by the probing packet. we pick up the packet pair. This is because the packet pair does not need a lot of packet transmission and does not need large size packet transmission.

\subsection{Path selection for traffic distribution}

\section{- Packet-by-packet path selection} HA must pick up one of available paths, whenever it receives the IP packet. The selection of path can be aware of TCP/UDP session or unaware of. Since it is hard to predict the actual traffic pattern and volume for each TCP/UDP session, we propose to pick up session un-aware path selection. It means the transmission path is selected packet-by-packet.

- Allocation of bandwidth for each path

Figure 3 shows the example how the bandwidth is allocated to each path. First, the whole of bandwidth is equally divided among the MNs, which is excepting the MN without the reception of ACK message against packet pair probing. MN1 and MN2 
in the figure has just registered to HA, but have not completed the bandwidth measurement. MN5 does not respond to the packet pair probing. Second, the allocated bandwidth for the path with completed bandwidth measurement is re-divided in proportion to the measured bandwidth for each path.

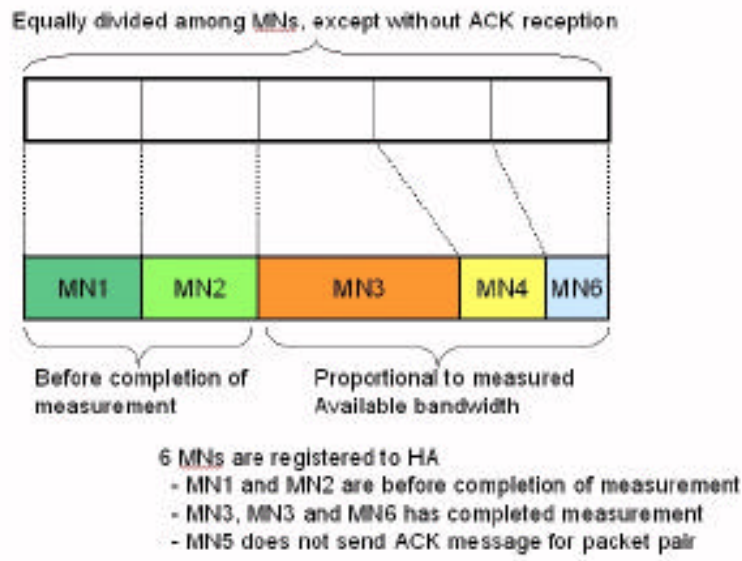

Figure 3 Allocation of bandwidth for each path

\section{Implementation of Prototype and Performance Evaluation}

In this section, we describe the prototype implementation and it's performance evaluation.

\subsection{Prototype System Implementation}

As discussed in the previous section, we use the packet pair for bandwidth measurement. We use the "bing" software [11]. "bing" uses ping messages with different size of ICMP packet to the target two nodes. HA and MN are implemented on Vine Linux 2.5.

\subsection{Evaluation System}

Figure 4 shows the evaluation system. We do not use actual wireless link for MN. The wireless links between Router to MN1 and Router to MN1 is emulated by the wired link applying the "dymmynet". By applying the dummynet at Router, MN1 and MN2, the available bandwidth for these links can be controlled, so as to fluctuate their available bandwidth.

\subsection{Evaluation Results}

Figure 5 shows the performance evaluation, associated with the effective end-to-end throughout using SCP (Secure Copy Protocol). Using the dummynet, the link bitrate for MN1 and MN2 is set as below. Here, in the evaluation, we have not change the available bandwidth for these links.
- $\quad$ Bit-rate for access link for MN

$>\mathrm{MN} 1: 128 \mathrm{kbps}$

$>\quad$ MN2 : $576 \mathrm{kbps}$

- $\quad$ File size to be transmitted from $\mathrm{CN}$ to $\mathrm{MN1}$

$1 \mathrm{MB}, 3 \mathrm{MB}, 5 \mathrm{MB}, 10 \mathrm{MB}$

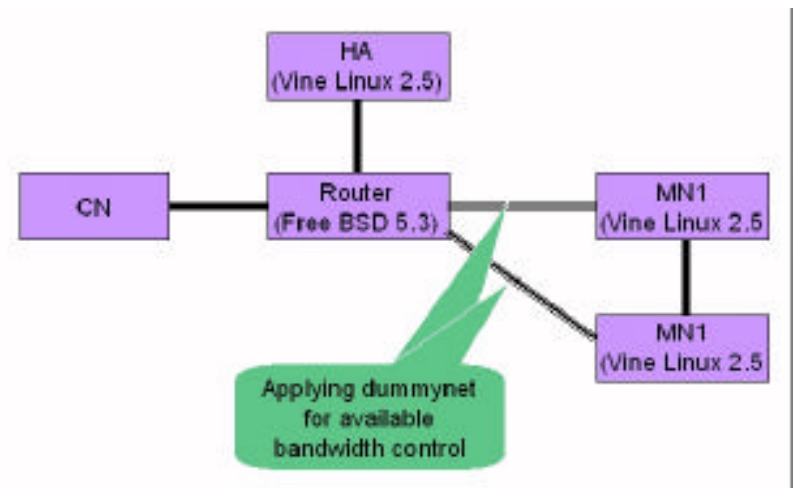

Figure 4 Evaluation system

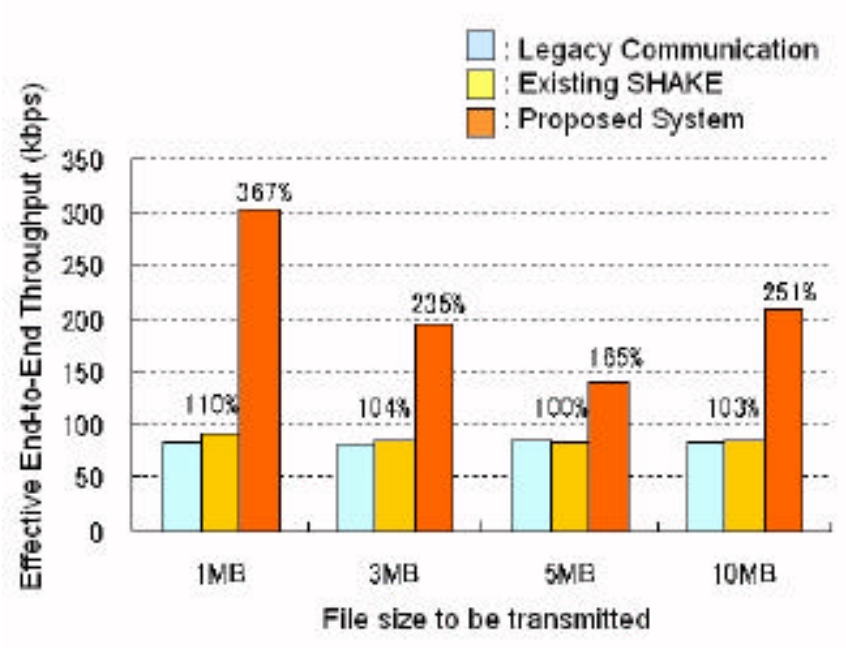

Figure 5 Result of Performance Evaluation

\section{Conclusion}

In this paper, the dynamic traffic balancing mechanism for SHAKE system with available bandwidth measurement is proposed and performed a preminary evaluation. The prototype system implementing the proposed mechanism can achieve large performance improvement.

SHAKE is the architecture using the mobile IP so as to share the multiple external cell-phone links via the wireless LAN link. The evaluation performed in this paper is just preminary. We must evaluate the proposed mechanism with more realistic condition, e.g., using the real cell-phone link or using the link whose available link is dynamically changed. 


\section{REFERENCES}

[1] Kenji Koyama, Yosuke Ito, Hiroshi Mineno, Susumu Ishihara, "Performance evaluation of TCP on Mobile IP SHAKE," IPSJ Journal, Vol.45, No.10, pp.2270-2278, Oct.2004.

[2] C. Perkins, "IP Mobility Support for IPv4", RFC3344, August 2002.

[3] R. Stewart, Q. Xie, K. Morneault, C. Sharp, H. Schwarzbauer, T. Taylor, I. Rytina, M. Kalla, L. Zhang, V. Paxson, "Stream Control Transmission Protocol", RFC2960, October 2000.

[4] Thomas J. Hacker, Brian D. Athey, "The End-to-End Performance Effects of Parallel TCP Sockets on a Lossy Wide-Area Network", Proceedings of the 16th IEEECS/ACM International Parallel and Distributed Processing Symposium, 2002.

[5] K. Rojviboonchai, H. Aida, "An Evaluation of Multi-path Transmission Control Protocol (M/TCP) with Robust Acknowledgement Schemes", Internet Conference 2002, October, 2002.

[6] C. Perkins., "IP Encapsulation within IP", RFC2003, October 1996.

[7] S.Uda, N.Ogashiwa, K,nagami, K.Kondo, I.Nakagawa, Y.Shinoda, H.Esaki, "Design and Implementation of Overlaying Multi-Homing Architecture", Workshop on IPv6 and Application at IEEE/IPSJ SAINT2004, January 2004.

[8] H.Esaki, "Multi-Homing and Multi-Path Architecture Using Mobile IP and NEMO Framework", Panel 1 (The Future of the Internet Architecture) at IEEE/IPSJ SAINT2004, January 2004.

[9] K.nagami, S.Uda, N.Ogashiwa, R.Wakikawa, H.Esaki, H.Onishi, "Multi-homing for small scale fixed network Using Mobile IP and NEMO", (work-in-progress), IETF Internet-Draft, draft-nagami-mip6-nemo-multihome-fixednetwork-03.txt, Feburary 2005.

[10] V.Devarapalli, R.Wakikawa, A.Petrescu, P.Thubert, "Network Mobility (NEMO) Basic Support Protocol", IETF RFC3963, January 2005.

[11] Satellite Link Simulation, http://ai3.asti.dost.gov.ph/sat/index.html 